\title{
Development of Single Phase Feeding Power Conditioner for Shinkansen Depots
}

\author{
Yoshifumi MOCHINAGA \\ General Manager, Power Supply Technology Development Div. \\ Tetsuo UZUKA \\ Senior Engineer, Power Supply Systems G.
}

\begin{abstract}
The feeding transformer for AC electric railways converts three-phase electric power into a pair of single-phase electric power supplies for driving electric cars. To prevent the fluctuation of three-phase voltage, however, a single phase feeding suits car depots. Authors have developed a single-phase feeding power conditioner (SFC) for unbalanced voltage compensation. This paper describes the principle and test results of using SFC. SFC is made of a scalene Scott-connected feeding transformer and a pair of self-commutated inverters, which connect the secondary side of a transformer and are coupled with the DC side capacitor. Each inverter operates independently in order to control reactive power. On the other hand, SFC controls active power from larger to small load phase circuits via the DC capacitor to keep the active power balanced.
\end{abstract}

Keywords : AC electrification, substation, power electronics, single phase feeding power conditioner

\section{Introduction}

The power of AC feeding electric railways, such as the Shinkansen of JR group, must be received from a powerful and high-tension source. At railway substations of main lines in Japan, the feeding transformer transfers three-phase electric power to a pair of single-phase feeding circuits.

But in some cases, a three-phase to single-phase feeding system may be better than a three-phase to 2-phase system for the following reasons, for example.

1) In the different phase feeding of directional system, trains have to run across different-phase circuits at every substation (and sometimes at every sectioning post.)

2) The directional feeding system doesn't suit depots that need single phase feeding.

We are trying to apply a new type of three-phase to single-phase transformation feeding system called the single phase feeding power conditioner (SFC). A scalene Scott-connected transformer and a pair of self-commutated inverters constitute a basic configuration of SFC. SFC has been now in commercial use at the Nagano Shinkansen depot since October 1997.

This paper gives outlines of the SFC and the theoretical studies of compensation using an SFC, and reports the successful results of small models used for all traction systems.

\section{Single-phase load and Scalene Scott-connected transformer}

\subsection{Balance with Scott-connected transformer}

\subsubsection{Feeding transformer}

If we obtain a large quantity of single-phase power from a three-phase power grid, three-phase unbalance will occur. In Japan, the voltage unbalance ratio, which is defined as the ratio of three-phase negative phase sequence voltage to positive phase sequence voltage must be held below $3 \%$ under the average load of two continuous hours on AC electrified lines.

The Scott-connected feeding transformer (See Fig. 1) can save the unbalance on the three-phase side. Equation (1) shows the relationship between primary and secondary currents of Scott-connected feeding transformer.

$\left.\begin{array}{l}\dot{I}_{\mathrm{U}}=\dot{I}_{\mathrm{M}}-\dot{I}_{\mathrm{T}} / \sqrt{3} \\ \dot{I}_{\mathrm{V}}=2 \dot{I}_{\mathrm{T}} / \sqrt{3} \\ \dot{I}_{\mathrm{W}}=-\dot{I}_{\mathrm{M}}-\dot{I}_{\mathrm{T}} / \sqrt{3}\end{array}\right\}$

If the load of the main phase is equal to that of the teaser, all the three phase currents become the same scale.

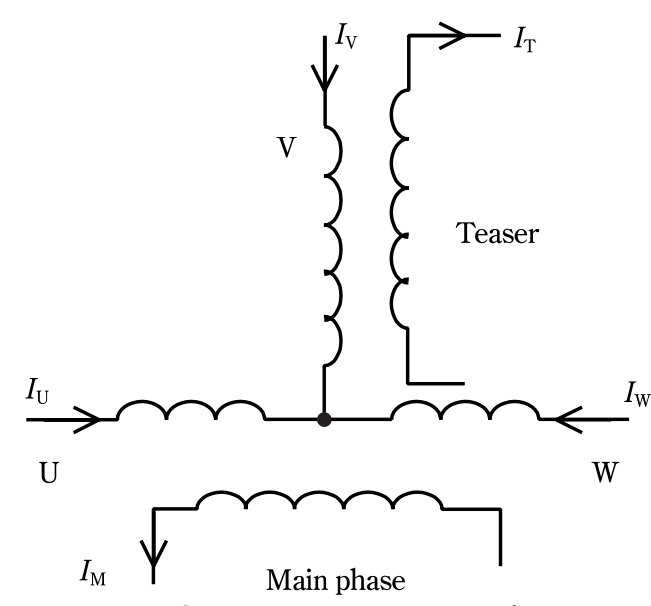

Fig. 1 Scott-connected transformer 


\subsubsection{Voltage fluctuation}

Equation (2) shows the voltage fluctuation of each of the three phases caused by the Scott-connected feeding transformer. Where $\gamma$ is the impedance angle of a power grid.

$$
\left.\begin{array}{l}
\Delta V_{\mathrm{UV}}=X\left\{I_{\mathrm{M}} \sin \left(\theta_{\mathrm{M}}-\gamma+5 \pi / 6\right)+\sqrt{3} I_{\mathrm{T}} \sin \left(\theta_{\mathrm{T}}-\gamma+\pi / 3\right)\right\} \\
\Delta V_{\mathrm{VW}}=X\left\{I_{\mathrm{M}} \sin \left(\theta_{\mathrm{M}}-\gamma+\pi / 6\right)+\sqrt{3} I_{\mathrm{T}} \sin \left(\theta_{\mathrm{T}}-\gamma+2 \pi / 3\right)\right\} \\
\Delta V_{\mathrm{WU}}=X\left\{2 I_{\mathrm{M}} \sin \left(\theta_{\mathrm{M}}-\gamma+\pi / 2\right)\right\}
\end{array}\right\}
$$

The resistance of impedance of a power grid can be negligible. If the case when the main phase and teaser loads are the same and the power factor of the two loads is 1.0 , the voltage fluctuation caused by reactance becomes zero as shown in the following equations.

$$
\left.\begin{array}{l}
\Delta V_{\mathrm{UV}}=X\left\{I_{\mathrm{M}} \sin (\pi / 3)+\sqrt{3} I_{\mathrm{T}} \sin (-\pi / 6)\right\}=0 \\
\Delta V_{\mathrm{vW}}=X\left\{I_{\mathrm{M}} \sin (-\pi / 3)+\sqrt{3} I_{\mathrm{T}} \sin (\pi / 6)\right\}=0 \\
\Delta V_{\mathrm{wU}}=X\left(2 I_{\mathrm{M}} \sin 0\right)=0
\end{array}\right\}
$$

\subsection{Scalene Scott-connected transformer}

Fig. 2 shows an example of three-phase grid and scalene Scott-connected transformer that feeds traction loads from slant phase (from Teaser to Main-phase). The Scott angle (slant phase to Teaser) would express a transformation ratio between the main-phase and the teaser. A proper Scott angle for PWM controlled traction loads is $\pi / 4$ (45 degrees, lagged from the teaser). Then the equation (4) shows currents of the three-phase side caused by the single-phase load fed by Slant phase (when the V phase is a reference).

Where $\phi$ is the Scott angle from slant phase to teaser, and $\theta$ is the power factor angle of load.

$$
\left.\begin{array}{l}
\dot{I}_{\mathrm{U}}=-\dot{I}_{\sin }\left(\frac{\pi}{4}\right) \exp \left(-\frac{\mathrm{j} \pi}{4}\right)-\frac{\dot{I}}{\sqrt{3}} \cos \left(\frac{\pi}{4}\right) \exp \left(-\frac{\mathrm{j} \pi}{4}\right) \\
\dot{I}_{\mathrm{V}}=\frac{2 \dot{I}}{\sqrt{3}} \cos \left(\frac{\pi}{4}\right) \exp \left(-\frac{\mathrm{j} \pi}{4}\right) \\
\dot{I}_{\mathrm{V}}=\dot{I}_{\mathrm{s} i n}\left(\frac{\pi}{4}\right) \exp \left(-\frac{\mathrm{j} \pi}{4}\right)-\frac{\dot{I}}{\sqrt{3}} \cos \left(\frac{\pi}{4}\right) \exp \left(-\frac{\mathrm{j} \pi}{4}\right)
\end{array}\right\}
$$

After some manipulation, Equation 4 can be rewritten as Equation 5. These equations show that scales of currents $\mathrm{U}, \mathrm{V}$, and $\mathrm{W}$ become smaller in this order. Thus we must compensate for the main phase to be equal to the teaser load.

$$
\left.\begin{array}{l}
\dot{I}_{\mathrm{U}}=\left(-1-\frac{1}{\sqrt{3}}\right) \frac{\dot{I}}{\sqrt{2}} \exp \left(-\frac{\mathrm{j} \pi}{4}\right) \\
\dot{I}_{\mathrm{V}}=\frac{2}{\sqrt{3}} \times \frac{\dot{I}}{\sqrt{2}} \exp \left(-\frac{\mathrm{j} \pi}{4}\right) \\
\dot{I}_{\mathrm{w}}=\left(1-\frac{1}{\sqrt{3}}\right) \frac{\dot{I}}{\sqrt{2}} \exp \left(-\frac{\mathrm{j} \pi}{4}\right)
\end{array}\right\}
$$

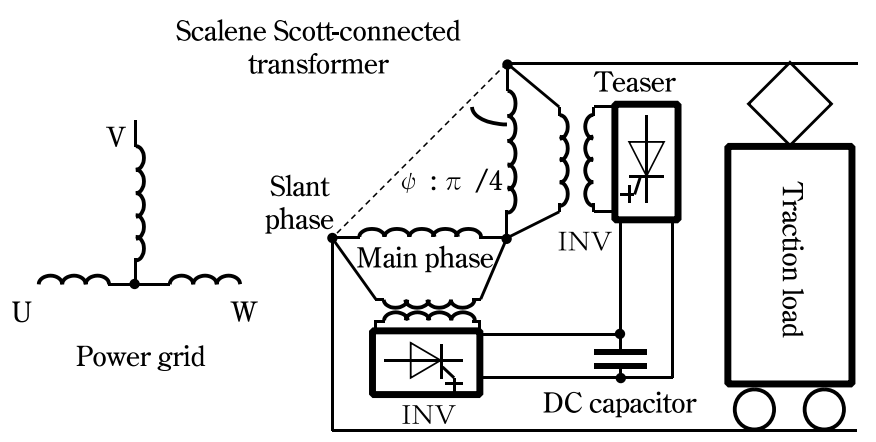

Fig. 2 Scalene Scott-connected transformer and SFC

\section{Principle compensate theory of SFC}

\subsection{Principle method of compensation}

An SFC is made of a pair of self-commutated inverters, which connect the two phases of secondary side of feeding transformer, coupled with the DC side capacitor. Fig. 2 shows the basic configuration of SFC.

Initially, to control reactive power, inverters operate independently at both the Main-phase side and the Teaser. Next, the power delivery control circuit compares the active power of the main-phase load and teaser load, and delivers active power from the circuit of the larger load to that of the smaller load via the DC capacitor. Then SFC can keep a balance of active power between the Main-phase circuit and the Teaser.

In the scalene Scott-connected transformer, the singlephase trainloads are regarded as a lead load in the main phase, and lag load in teaser.

All control circuits that control active power passing, DC voltage fluctuation and reactive power compensation issue orders to their respective objects. And current control circuit integrates these orders and decides the current object of inverters. The PWM control circuit drives GTO thyristors of the inverters for the current object.

The SFC feeds trains from a slant phase (from Teaser to Main-phase) of the secondary side of the scalene Scottconnected transformer. A pair of self-commutated inverters is controlled by the PWM method so that they can handle active power and reactive power. Inverters compensate reactive power of each secondary side to regulate three-phase voltage fluctuation. Furthermore, the inverters connected back-to-back on the DC side can mutually accommodate the active power in the main-phase and teaser of secondary side of transformer, so that the threephase side active power is balanced.

\subsection{Principle method to compensate}

Set a traction load in the slant phase feeding circuit of SFC (Scott angle is $\pi / 4$, see Fig. 3 ), where $\theta$ is the power factor angle of loads; $P$ is the active power, $Q$ is the reactive power, $I$ is the load current, and $V_{\mathrm{L}}$ is the feeding voltage (slant phase). Then the secondary side active and reactive power $P_{\mathrm{M}}, P_{\mathrm{T}}, Q_{\mathrm{M}}, Q_{\mathrm{T}}$ become as shown by Equa- 
tion (6). Let's set the power of load $W$ as $W=V_{\mathrm{L}} I$, for simplification.

$$
\left.\begin{array}{l}
P_{\mathrm{M}}=V_{\mathrm{L}} \cos \left(\frac{\pi}{4}\right) \times I \cos \left(\frac{\pi}{4}-\theta\right)=0.5 W(\cos \theta+\sin \theta) \\
P_{\mathrm{T}}=V_{\mathrm{L}} \sin \left(\frac{\pi}{4}\right) \times I \cos \left(\frac{\pi}{4}+\theta\right)=0.5 W(\cos \theta-\sin \theta) \\
Q_{\mathrm{M}}=-V_{\mathrm{L}} \cos \left(\frac{\pi}{4}\right) \times I \sin \left(\frac{\pi}{4}-\theta\right)=-0.5 W(\cos \theta-\sin \theta) \\
Q_{\mathrm{T}}=V_{\mathrm{L}} \cos \left(\frac{\pi}{4}\right) \times I \sin \left(\frac{\pi}{4}+\theta\right)=0.5 W(\cos \theta+\sin \theta)
\end{array}\right\}
$$

To reduce three-phase voltage fluctuation, SFC reduces the reactive power of main-phase and teaser $\left(Q_{\mathrm{M}}\right.$, $\left.Q_{\mathrm{T}}\right)$ to zero. SFC always provides the active power of Mainphase and Teaser $\left(P_{\mathrm{M}}, P_{\mathrm{T}}\right)$ to balance three-phase power. The active power $P_{\mathrm{C}}$ provided from main-phase $P_{\mathrm{M}}$ to teaser $P_{\mathrm{T}}$ is an average of the power on both sides, expressed by the next equation.

$$
P_{\mathrm{C}}=\left(P_{\mathrm{M}}-P_{\mathrm{T}}\right) / 2=0.5 W \sin \theta
$$

In this way SFC divides the single-phase load into a pair of single-phase loads, and three-phase side currents should be balanced and voltage fluctuation would be minimized. Table 1 shows the relationship between the type of load and SFC mode. In general, the running load is larger than the regenerative load.

Table 1 Type of load and SFC mode

\begin{tabular}{|l|l|l|}
\hline Type of load & Main phase $Q_{\mathrm{Mc}}$ & Teaser $Q_{\mathrm{Tc}}$ \\
\hline Running & Reactor & Capacitor \\
\hline Regenerative & Capacitor & Reactor \\
\hline
\end{tabular}

\subsubsection{For PWM controlled car}

Fig. 3(b) shows a case of compensating for PWM controlled car running. The load's power factor angle is 0 , and SFC minimizes the reactive power of main-phase and teaser $\left(Q_{\mathrm{M}}, Q_{\mathrm{T}}\right)$. Equation (8) shows the reactive power to compensate. Equation (9) also shows the active power in this situation. SFC doesn't have to handle the active power to balance the three-phase power. Equation (10) shows a summation of active power that is equal to the load power capacity $W$.

$$
\left.\begin{array}{l}
Q_{\mathrm{MC}}=V_{\mathrm{L}} \cos \left(\frac{\pi}{4}\right) \times I \sin \left(\frac{\pi}{4}\right)=0.5 V_{\mathrm{L}} I=0.5 W(\text { Reactor }) \\
Q_{\mathrm{TC}}=-V_{\mathrm{L}} \cos \left(\frac{\pi}{4}\right) \times I \sin \left(\frac{\pi}{4}\right)=-0.5 V_{\mathrm{L}} I=-0.5 W(\text { Capacitor })
\end{array}\right\}
$$

\subsubsection{For thyristor phase controlled car}

Fig. 3(c) shows a case of Thyristor phase controlled car $(\theta=\pi / 6)$. In this case, SFC compensates the reactive power of main-phase and teaser $\left(Q_{\mathrm{M}}, Q_{\mathrm{T}}\right)$, first as shown by Equation (11). Then, SFC will accommodate the active power $P_{\mathrm{C}}$ provided from main-phase $P_{\mathrm{M}}$ to teaser $P_{\mathrm{T}}$, since $P_{\mathrm{M}}>P_{\mathrm{T}}$ as in Equation (12).

$$
\left.\begin{array}{l}
Q_{\mathrm{MC}}=-V_{\mathrm{L}} \cos \left(\frac{\pi}{4}\right) \times I \sin \left(\frac{\pi}{4}-\frac{\pi}{6}\right)=0.183 V_{\mathrm{L}} I=0.183 W(\text { Reactor }) \\
Q_{\mathrm{TC}}=V_{\mathrm{L}} \sin \left(\frac{\pi}{4}\right) \times I \sin \left(\frac{\pi}{4}+\frac{\pi}{6}\right)=-0.683 V_{\mathrm{L}} I=-0.683 W(\text { Capacitor })
\end{array}\right\}
$$

The quantity of active power to accommodate is shown by Equation (13). In this method, active power of main phase and teaser become equal.

$$
\left.\begin{array}{l}
P_{\mathrm{M}}{ }^{\prime}=(0.683-0.25) W=0.433 W \\
P_{\mathrm{T}}{ }^{\prime}=(0.183+0.25) W=0.433 W \\
P=P_{\mathrm{M}}{ }^{\prime}+P_{\mathrm{T}}{ }^{\prime}=0.866 W
\end{array}\right\}
$$$$
P_{\mathrm{dc}}=\left(P_{\mathrm{M}}-P_{\mathrm{T}}\right) / 2=0.25 W
$$

\section{Small model of SFC}

\subsection{Outline}

\subsubsection{Circuits and Composition}

We made a mock-up of the SFC to estimate our principle theory. The model system consists of a pair of self-

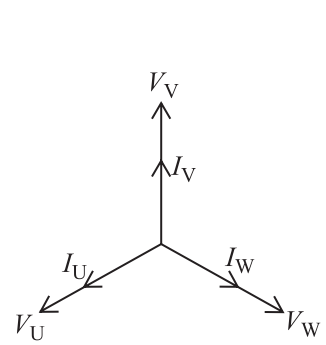

(a) Three-phase side

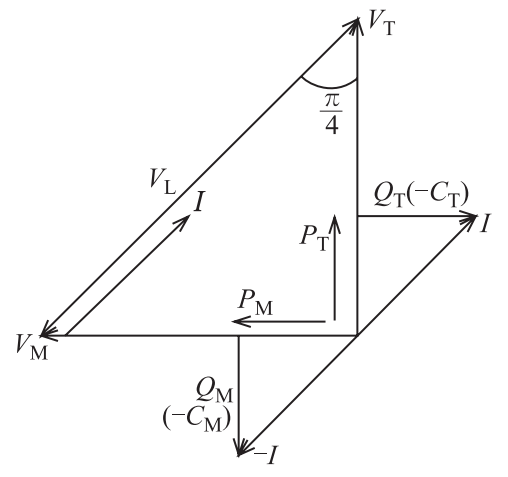

(b) PWM converter car

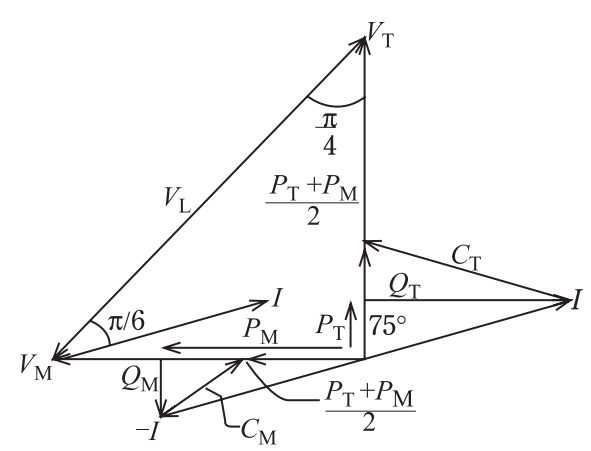

(c) Thyristor phase control car

Fig. 3 Philosophy of compensation 
commutated inverters, a scalene Scott-connected transformer, line inductors, an autotransformer, and a singlephase dummy load for a slant phase feeding circuit. One model simulates PWM-controlled cars, and the other simulates thyristor phase controlled cars. The output voltage of the single inverter is $220 \mathrm{~V}$, and its capacity is $10 \mathrm{kVA}$. We use 1S-1P BiMOS power transistors to switch at a frequency of $7 \mathrm{kHz}$. It can simulate the real scale model using GTO thyristors with 3-pulse, 8-level series coupled.

\subsubsection{Control method}

The measured voltages and currents of main phase and teaser let the SFC inverters work to make the phase factor 1 and balance the active power. If the rated capacity of $\mathrm{SFC}$ is not enough to complete compensation, we control SFC as follows. We denote a rated capacity of SFC (main phase and teaser added) as $C_{M A X}$ and each phase capacity as $0.5 C_{M A X}$.

$$
C_{\text {MAX }}=k W(k: \text { compensation ratio })
$$

Then for the control method in the phase, which requires a larger capacity, set $k$ as equation (15).

$$
\text { Rated Capacity }=k \sqrt{{P_{\mathrm{dc}}{ }^{2}+Q_{\mathrm{TC}}{ }^{2}}^{2}}
$$

In such a situation where SFC has an insufficient capacity, we can predict three phase side currents and voltage fluctuations from these equations.

\subsection{Test result}

\subsubsection{Test circuit}

Fig. 4 shows an SFC model configuration. With this circuit, we can examine the basic active power delivery of main-phase and teaser, and compensation of reactive power. To adjust the slant phase voltage to about $200 \mathrm{~V}$, we set the autotransformer to Slant phase by using a dummy load.

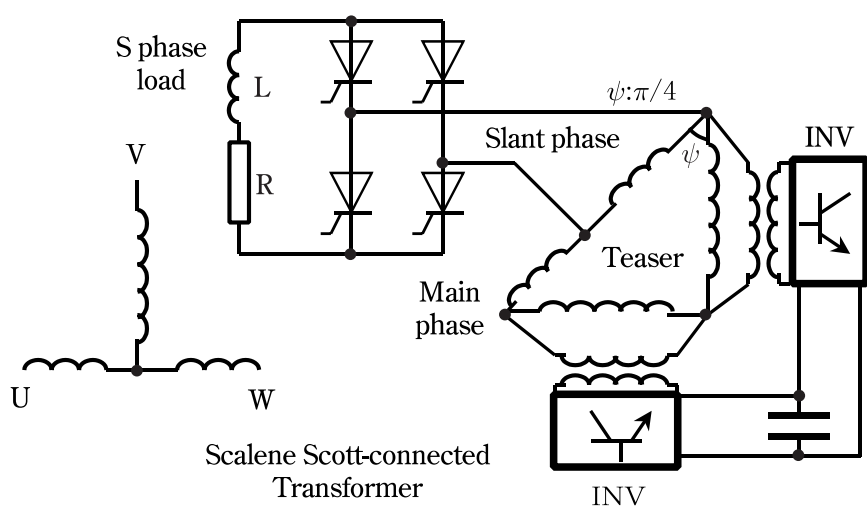

Fig. 4 SFC Test circuit

\subsubsection{Voltages and Currents of Test Circuit}

A result of SFC compensation, under the condition of slant phase where the thyristor phase control dummy load is set, is expressed in Fig. 5. Where the three-phase side current is in the order of $I_{\mathrm{U}}>I_{\mathrm{V}}>I_{\mathrm{W}}$ and unbalanced without compensation (a). Currents of Scalene Scott-connected transformer secondary side $I_{\mathrm{m}}$ and $I_{\mathrm{t}}$ are nearly equal since inverters did not operate.

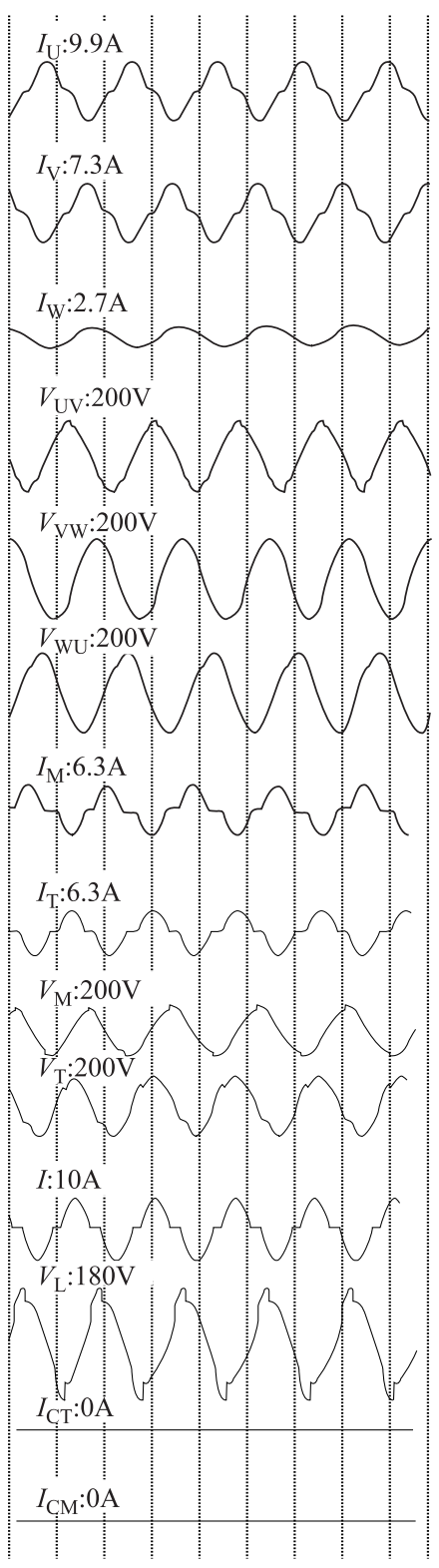

(a) Without compensation

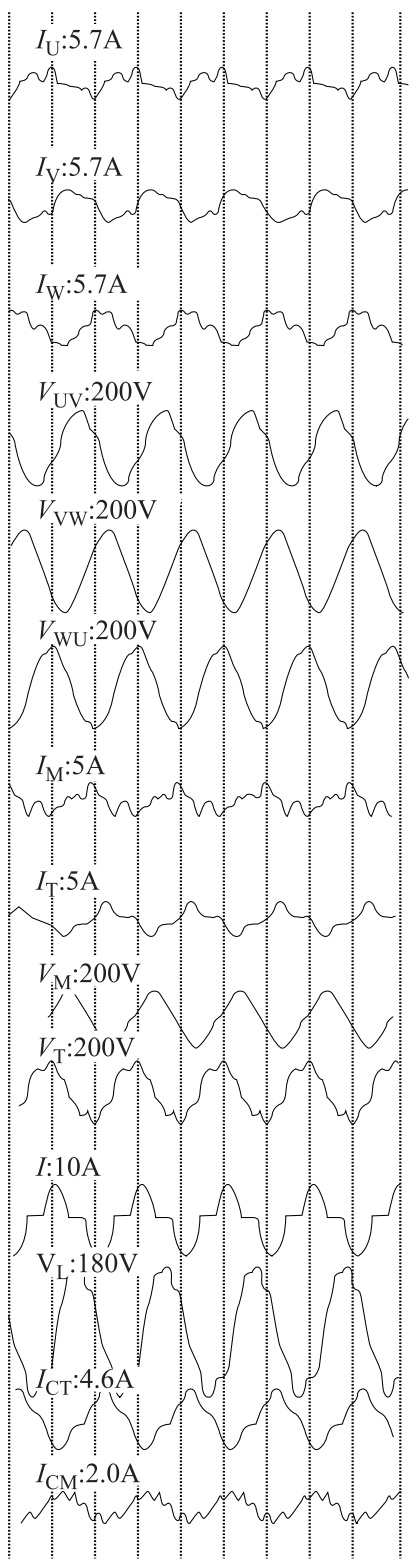

(b) With compensation
Fig. 5 voltages and currents

When the inverters operate, the three-phase current is approximately balanced by suitable reactive power compensation (b). $I_{\mathrm{m}}$ and $I_{\mathrm{t}}$ are also nearly equal, but smaller than in the case (a).

Fig. 6 shows the three-phase side current provided for the thyristor phase control load. In this case, currents are unbalanced more than resistor load. $I_{\mathrm{m}}$ and $I_{\mathrm{t}}$ are nearly equal. After SFC operation, currents of three-phase side are controlled to balance. $I_{\mathrm{m}}$ and $I_{\mathrm{t}}$ are nearly equal but smaller than in the case (a).

\subsubsection{Balancing Test}

The voltage unbalance ratio and voltage drop ratio (UV phase) for resistor and thyristor are illustrated in Fig. 6. As a result of compensation, the unbalance ratio dropped to a half. The unbalance between load and inverters supposedly cause this insufficient result. 


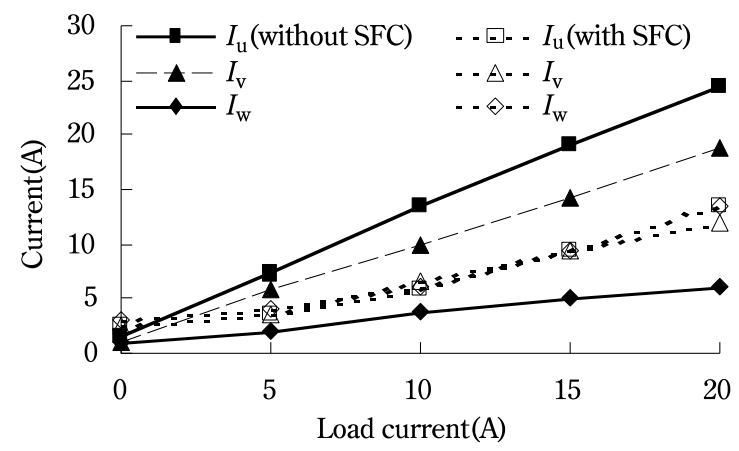

(a) Three-phase currents

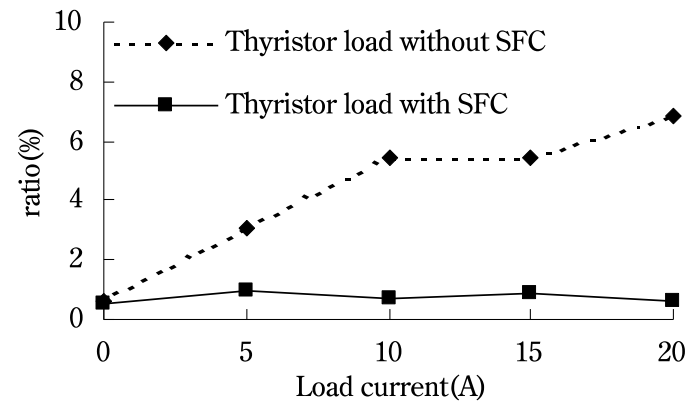

(b) Voltage unbalance ratio

Fig. 6 SFC's compensation: three-phase side data

\section{Conclusions}

We proposed an SFC that can feed various types of single-phase AC traction loads without three-phase side voltage fluctuation and unbalance, using inverters to compensate reactive power and accommodate the active power. We made a small model of SFC and proven principle compensating theory.

We thank Mitsubishi Electric Corporation who made our mock-up of the SFC.

We also thank deeply JR East, Meidensha and JRCC for commercial operation at the Nagano depot substation, Hokuriku Shinkansen.

\section{References}

1) Uzuka, Mochinaga, Hase, Yoshida: "Principle theory of Static Unbalanced Power Compensator with Selfcommutated inverters (in Japanese)", IEE of Japan, No. 1251, 1996

2) Mochinaga, Hase, Akita, Inoue, and Ohbe: "Development of Static Unbalanced Power Compensator for Nagano Shinkansen Depot (in Japanese)", IEE of Japan, Vol. 110-D, TER-97-45, 1997.12 